\title{
Nonatheosclerotic Giant Right Coronary Artery Aneurysm
}

\author{
Lucas Cardoso $^{1}$, Ricardo Dias ${ }^{1}$, Lucas Molinari Veloso da Silveira ${ }^{1}$, Lea Demarchi ${ }^{1}$, \\ Charles Mady $^{1}$, and Fábio Jatene ${ }^{1}$ \\ ${ }^{1}$ University of Sao Paulo Heart Institute
}

September 17, 2020

\begin{abstract}
We present an unusual case of a 67-year-old woman with an incidental finding of a cardiac mass on a chest-computed tomography. Coronary angiotomography confirmed the diagnosis of a right coronary artery aneurysm, with $5.7 \times 5.7 \mathrm{~cm}$. Patient underwent aneurysm resection and coronary artery bypass graft surgery, with subsequent histologic study suggestive of arteritis sequela. Patients with giant coronary artery aneurysms are at high risk of complications and must benefit from aneurysm exclusion. This is a rare condition that can also be part of a systemic inflammatory disease.
\end{abstract}

\section{CASE PRESENTATION}

A 67-year-old woman was referred to our hospital due to an incidental finding of a cardiac mass on a chestcomputed tomography (CT), during an investigation of sudden dyspnea. She had normal sinus rhythm, with no ST-segment changes on electrocardiography. Transthoracic echocardiography revealed a $6.3 \times 5.9 \mathrm{~cm}$ anechoic mass, partially compressing the right chambers; cardiac function was normal, and no ventricular akinesia was found. Coronary angiotomography showed a saccular aneurysmal dilatation in the middle segment of the right coronary artery (RCA), with $5.7 \times 5.7 \mathrm{~cm}$ associated with a calcified mural thrombus (Figure 1), without significant coronary stenosis. Investigation with whole-body CT scan and cerebral magnetic resonance imaging excluded other vessel aneurysms.

The patient underwent surgery through full sternotomy, with resection of a giant RCA aneurysm and exclusion of both entrance and exit ostia (Figure 2). Coronary artery bypass was performed with a saphenous vein graft to the RCA. Histologic study was suggestive of arteritis sequela represented by diffuse thickening of intimal and medial layers with fibrosis, and destruction of the elastic lamina (Figure 3).

Patients with giant coronary artery aneurysms are at high risk of complications and must benefit from aneurysm exclusion ${ }^{1}$. This is a rare condition and atherosclerosis accounts for half of the cases in adults; however, it may also be part of a systemic inflammatory disease ${ }^{2}$, as presented in this case.

\section{REFERENCES}

1. Pham V, Hemptinne Q, Grinda JM, et al. Giant coronary aneurysms, from diagnosis to treatment: A literature review. Arch Cardiovasc Dis. 2020;113(1):59-69.

2. Chia HMY, Tan KH, Jackson G. Non-atherosclerotic coronary artery aneurysms: Two case reports. Heart. 1997;78(6):613-616.

Figure 1. A-Coronary computed tomography angiography showing a saccular aneurysm with a partially calcified mural thrombus. B-3D reconstruction confirming that the mass was supplied by right coronary artery.

Figure 2. Intraoperative view of aneurysm exclusion. 
Figure 3. Photomicrograph of right coronary artery with sequelae of arteritis. Destruction of the elastic laminae (black color). I + M- intimal and medial layers; a- Coronary adventitia; L- Coronary lumen.

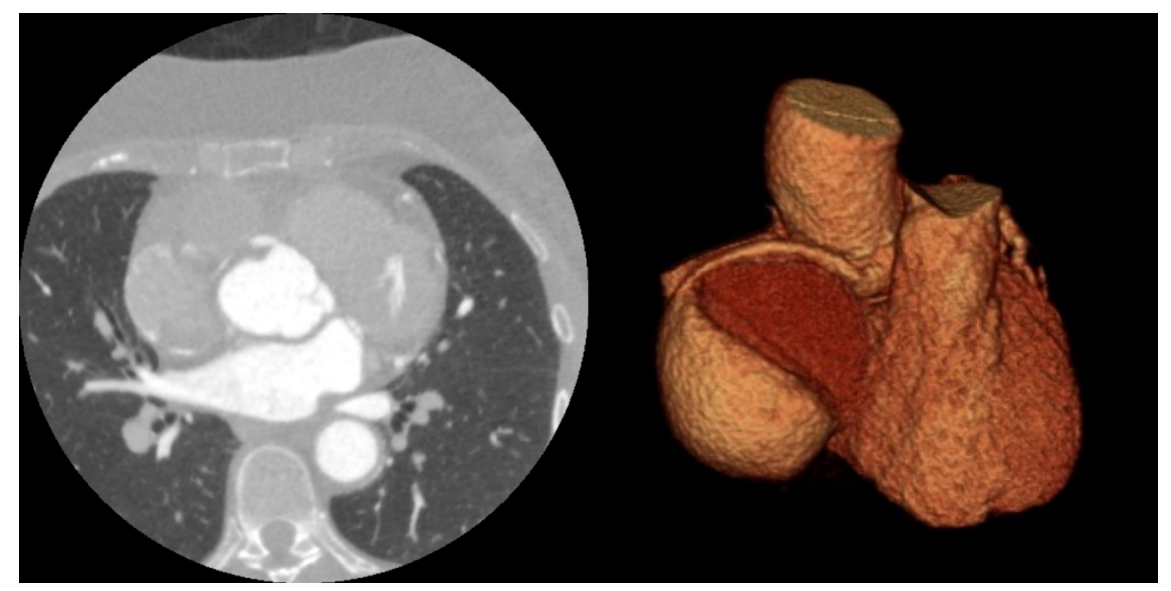




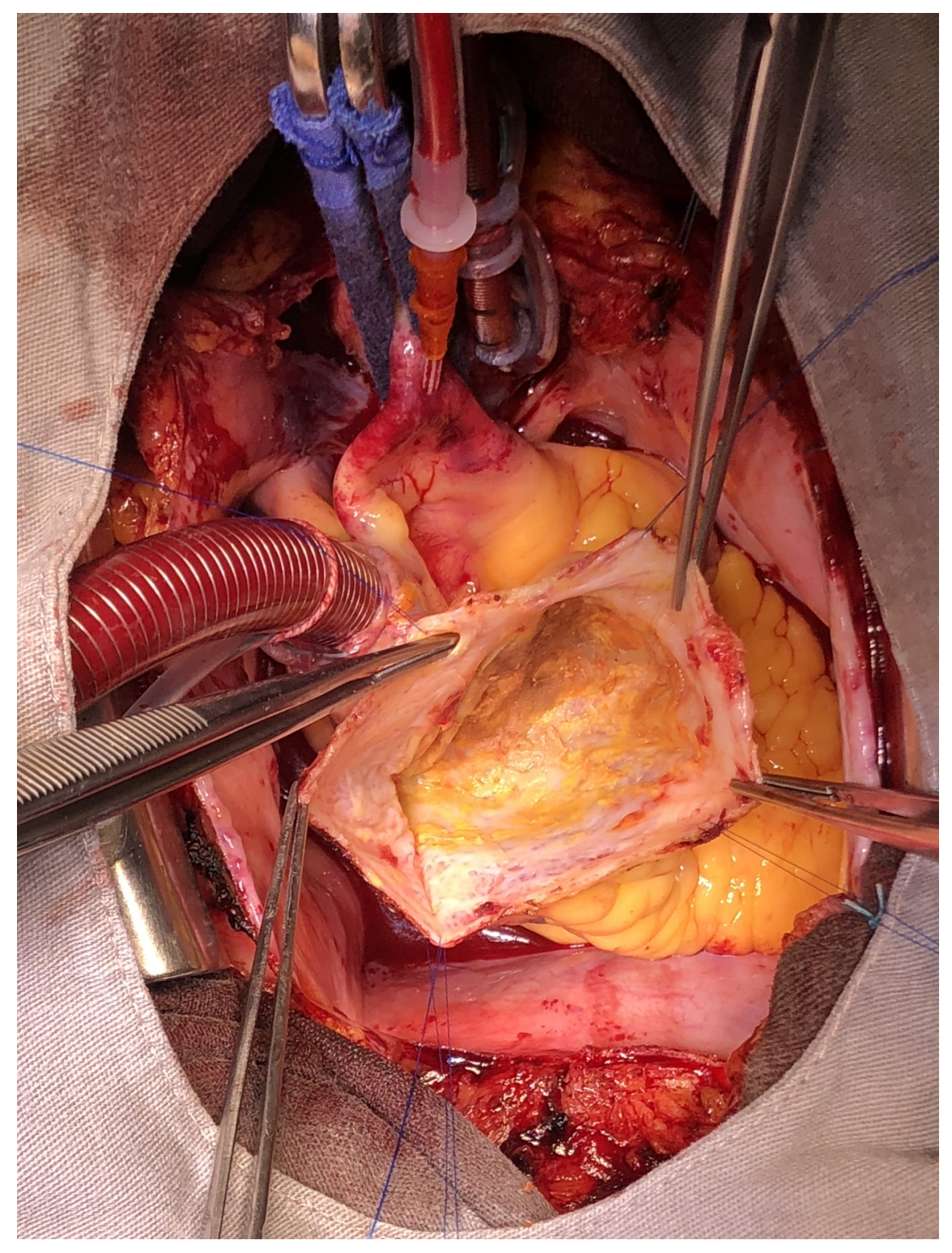




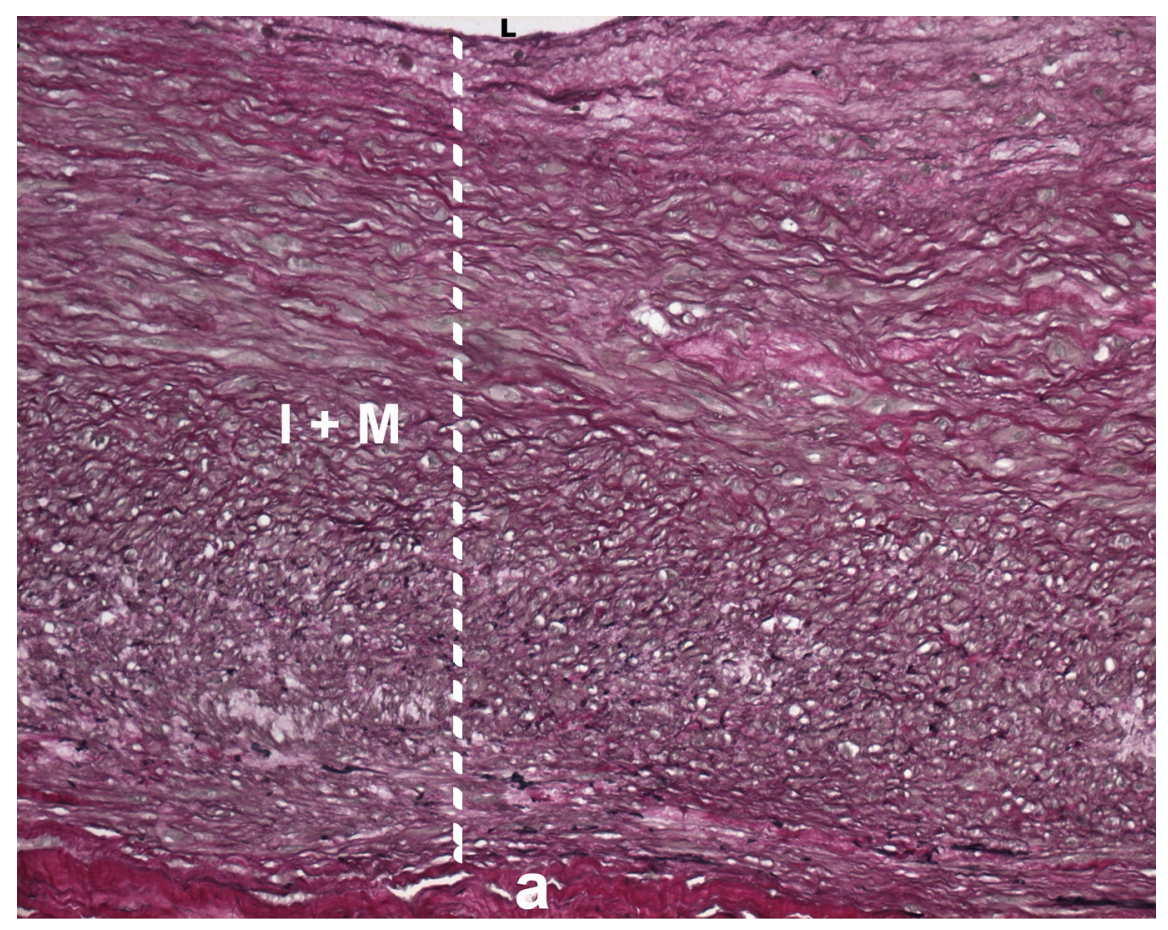

